\title{
Mullins effect in swollen rubber: Experimental investigation and constitutive modelling
}

\author{
A.B. Chai ${ }^{a}$, E. Verron ${ }^{\text {b }}$, A. Andriyana ${ }^{\mathrm{a}, *}$, M.R. Johan ${ }^{\mathrm{a}}$ \\ ${ }^{a}$ Department of Mechanical Engineering, University of Malaya, 50603 Kuala Lumpur, Malaysia \\ ${ }^{\mathrm{b}}$ LUNAM Université, École Centrale de Nantes, GeM, UMR CNRS 6183, BP92101, 44321 Nantes, France
}

\begin{abstract}
Apart from the well-known hyperelastic and large stress-strain behavior in dry rubber, the inelastic responses such as hysteresis and Mullins effect are also observed when a dry rubber is cyclically loaded. The former is given by different loading and unloading paths in a cycle, while the latter corresponds to the significant decrease in stress between two successive cycles, particularly between the first and second loading. The Mullins effect or the stress-softening effect disappears after several cycles of loading, i.e. five cycles for the materials used in the present study. A number of models describing the Mullins effect in dry rubber are available in the literature. Nevertheless, works focusing on the Mullins effect in swollen rubbers are less common. Therefore, the experimental investigation and modelling of Mullins effect in swollen rubbers are addressed in the present study. For this purpose, mechanical tests were conducted in order to probe the Mullins effect in swollen rubbers under cyclic loading conditions. Furthermore, the pseudo-elastic model [Ogden, R.W. \& Roxburgh, D. G., 1999. A pseudo-elastic model for the Mullins effect in filled rubber. Proc. Roy. Soc. A. 455, 2861-2877] is considered and extended in order to account for swelling level. Results show that the proposed model is qualitatively in good agreement with experimental observations.
\end{abstract}

\section{Introduction}

The world is facing an energy crisis and our environment is degrading due to excessive exploration and usage of fossil fuel. Hence, several proposals have been raised to overcome this circumstance. One of the feasible solutions is through the introduction of renewable energy, e.g. biodiesel. Biodiesel, which is derived from renewable resources such as animal fat and vegetable oil, is claimed to provide better energy efficiency and offer cleaner environment. However, utilizing such fuel in the existing engine systems creates several compatibility issues for the automotive components, particularly the rubber materials [1,2]. Rubber materials are known to experience swelling when in contact with the fuel

\footnotetext{
* Corresponding author. Tel.: +60 37967 5254; fax: +60 379675317.

E-mail address: andri.andriyana@um.edu.my (A. Andriyana).
}

and this will contribute to deterioration of their mechanical properties. In addition to the swelling, rubber components such as o-rings or gaskets in sealing systems are also subjected to mechanical loading during their service. Therefore, it is crucial to understand the mechanical response of the swollen rubber in order to develop durable and robust components and to predict their service life $[3,4]$.

Under static loading condition, it is well-known that rubber materials exhibit a highly non-linear stress-strain response. This response is time-dependent which can be demonstrated by relaxation and creep experiments. The hyperelasticity of the rubber material has been described using different strain energy functions and a large number of them are incorporated into commercial FEA codes. Note that the efficiency of these hyperelastic models is compared by Marckmann and Verron [5] in their review paper. In addition to the above response, when a dry rubber is subjected to cyclic loading, it exhibits strong inelastic 
responses such as hysteresis and stress-softening. The hysteresis is characterized by different loading and unloading paths and can be related to either viscoelasticity [6], viscoplasticity [7] or strain-induced crystallization [8]. The stress-softening corresponds to a significant decrease in stress between two successive cycles, particularly between the first and second loadings. This phenomenon was first observed by Bouasse and Carrière [9] and intensively studied by Mullins [10]. It is often referred to as the Mullins effect. While the Mullins effect is well-known in both filled and unfilled crystallizing dry rubbers [11], it is recently demonstrated that it is also observed in swollen rubbers $[12,13]$. As reviewed by Diani et al. [11], there are many efforts proposing different theories to explain the stresssoftening phenomenon in dry rubber. Nevertheless, to date there is no unanimous microlevel explanation for the stress-softening [11,14].

In contrast to dry rubbers, only a few studies dealing with observation of the Mullins effect in swollen rubbers and gels are available $[12,13,15,16]$. Moreover, corresponding constitutive models are not available in the literature. Hence, the objective of the present paper is to propose a simple phenomenological model to capture the Mullins effect observed in swollen rubbers under cyclic loading conditions. Other inelastic responses such as hysteresis and permanent set are not considered. The pseudo-elastic model of Ogden and Roxburgh [17] for the Mullins effect is modified and extended in order to account for the swelling level. The dependence of the Mullins effect on swelling is probed through mechanical testing of swollen rubbers. Two types of filled rubber are considered: Nitrile Butadiene Rubber (NBR) and Polychloroprene Rubber (CR). The experimental program is detailed in Section 2. The basic theory of pseudo-elastic model is briefly recalled in Section 3 and extended by considering the experimental observations. The efficiency of the model is assessed in Section 4. Finally, concluding remarks are given in Section 5.

\section{Experimental program}

\subsection{Materials}

Commercial grade NBR and CR materials with $60 \pm 5$ Shore hardness A were purchased from MAKA Engineering Sdn. Bhd., Malaysia. The NBR and CR have specific gravity of $1.4 \pm 0.1$ and $25 \mathrm{wt}$.\% of carbon black. The vulcanization of these two materials was performed by compression molding at $165{ }^{\circ} \mathrm{C}$ for $5 \mathrm{~min}$ under a pressure of approximately 6.89 $\mathrm{MPa}$ in an electrically heated press. Due to confidentiality constraint, the detailed compound ingredients are not provided here.

The palm biodiesel (B100) used as immersion medium in this research was provided by Am Biofuels Sdn. Bhd., Malaysia. The analysis report of the palm biodiesel used in the present study is available elsewhere [18]. To systematically characterize the effect of palm biodiesel on the mechanical responses of the selected elastomers, a standard Malaysian on-road diesel fuel (B0) was also used in this study.

The rubber specimens for swelling and mechanical tests were annular cylindrical blocks with outside diameter $=$ $50 \mathrm{~mm}$, inner diameter $=38 \mathrm{~mm}$ and height $=10 \mathrm{~mm}$.

\subsection{Swelling measurement}

The swelling of NBR and CR in diesel (B0) and biodiesel (B100) were measured from free swelling tests conducted at room temperature for various immersion times: 2, 5, 10, 20 and 30 days. Fig. 1 showed the simple immersion test where the specimens were hung and immersed in the stainless steel container containing biodiesel. A separate container containing diesel was used to compare the effect of different fuels on the swelling behavior of the rubbers. Each specimen was completely immersed in the tested fuel. After the desired immersion time, specimens were removed from the container and dipped quickly into acetone to remove the excess oil. The samples were then blotted dry with filter paper. The weight in air and in water for the specimen before and after immersion was measured using a balance. Note that for each immersion time, the average results were calculated from four test specimens. The volume change, $\varepsilon_{S}$ was then calculated from the following relation [2]:

$\varepsilon_{s}=\frac{\left(M_{2}-M_{4}\right)-\left(M_{1}-M_{3}\right)}{\left(M_{1}-M_{3}\right)}$.

where $M_{1}$ and $M_{2}$ are the mass in air (gram) before and after immersion while $M_{3}$ and $M_{4}$ are mass in water (gram) before and after immersion, respectively. The degree of swelling $J_{S}$ was calculated from the volume change using the following relation [19]:

$J_{s}=\frac{V}{V_{o}}=1+\varepsilon_{s}$.

where $V$ and $V_{o}$ are, respectively, the weight of swollen and dry rubbers.

\subsubsection{Swelling results}

The variation of degree of swelling of NBR and CR as a function of immersion time is shown in Fig. 2. The swelling in NBR and CR increases when the exposure time is increased from 2 days to 30 days. It is observed that for a given immersion time, the swelling of NBR and CR are higher in palm biodiesel than in diesel. The rate of swelling appears to be high at short exposure time before decreasing at longer exposure time. Rapid swelling is initiated with absorption of liquids when the liquids dissolve in the surface layer of the rubber (adsorption) until a certain concentration. Subsequently, the liquids penetrate slowly into the rubber by diffusion until the rubber specimen achieves equilibrium swelling [20]. Further discussion on the swelling of rubbers in palm biodiesel and diesel can be found, for example, in [1,13].

\subsection{Mechanical testing for Mullins effect measurement}

In order to probe the effect of swelling on the Mullins effect, uniaxial cyclic compressive tests were carried out on dry and swollen rubber specimens using an Instron test machine operated at room temperature. Circular compression plates were attached to the machine to ensure uniform displacement control on the specimens. The experimental setup was connected to a computer to record the experimental data. The tests were conducted at a constant displacement rate of $0.1 \mathrm{~mm} / \mathrm{s}$ to avoid excessive increase in 

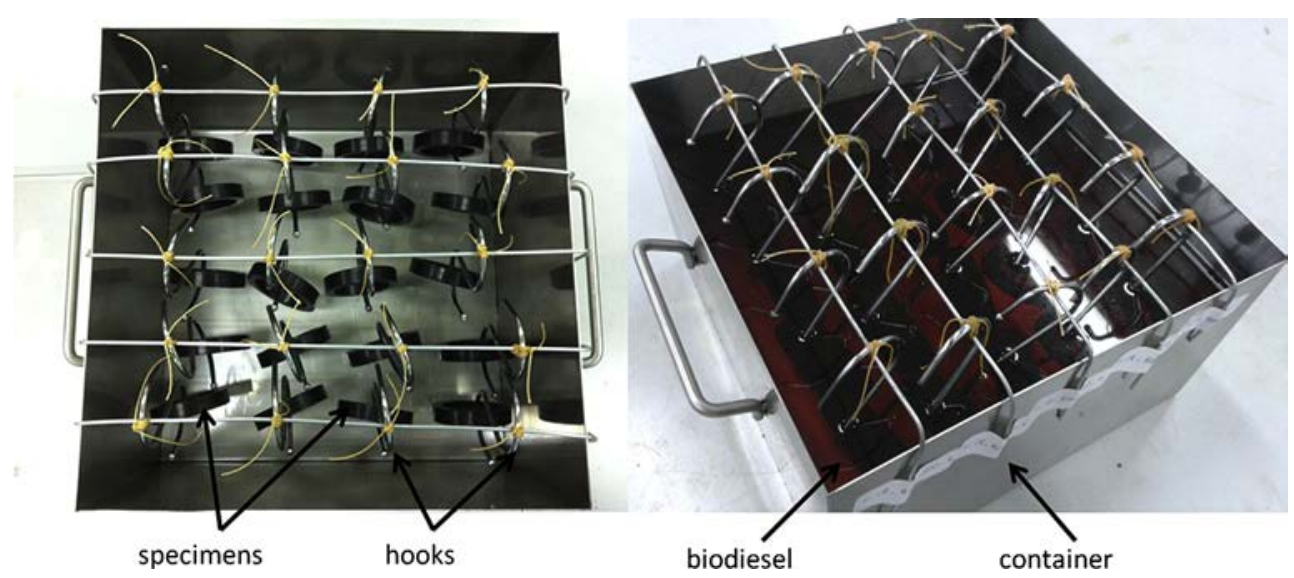

Fig. 1. Free swelling test for the specimens before immersion (left) and after immersed (right) in tested fuel (B100).

the temperature of the specimens, i.e. thermal effects are not considered in the present study. Cyclic compressive tests where the specimens were subjected to two maximum strain levels (30\% and $40 \%$ ) with 6 cycles at each strain were conducted. To reduce uncertainty of the results, at least four specimens were used in each test.

\subsubsection{Mullins effect results}

The stress-strain response of dry and swollen rubbers (after immersed in B0 and B100 for 30 days) under cyclic compressive loading is presented in Fig. 3. Generally, it is observed that the nature of the stress-strain curves is preserved. However, for a given strain, lower stresses are recorded for swollen rubbers. CR and NBR swollen by B100 show lower stress level than the corresponding rubbers swollen by B0. Both dry and swollen rubbers exhibit the Mullins effect which disappears after around five loading cycles. Moreover, it can be noted that for both NBR and CR, the stress softening appeared to be significantly smaller in the case of swollen rubbers. Furthermore, it is shown that the reloading paths do not rejoin the primary path at the maximum strain.

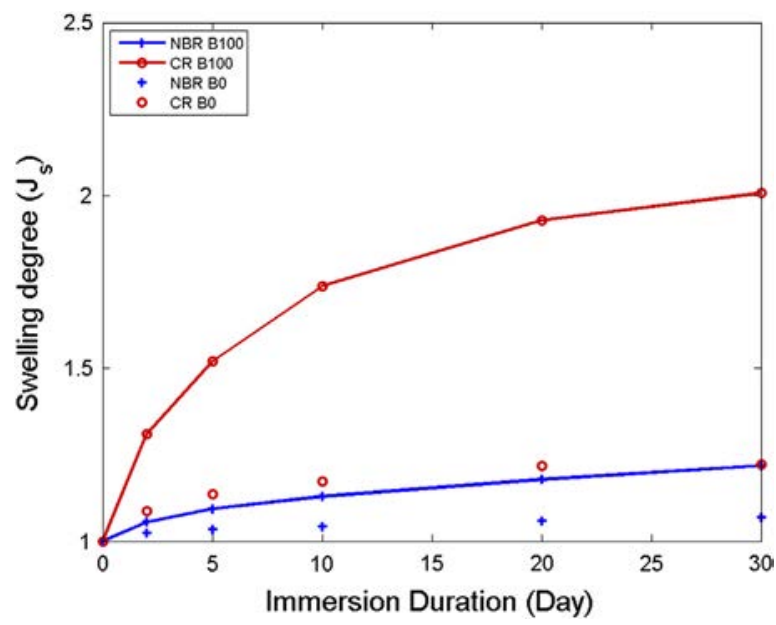

Fig. 2. Swelling degree $\left(J_{s}\right)$ exhibited by NBR and CR after immersion in palm biodiesel (B100) and diesel (B0) at different immersion durations.
As clearly shown in Fig. 3, in addition to the Mullins effect, both dry and swollen rubbers exhibit hysteresis and permanent set. Since the Mullins effect is the only inelastic response addressed in the present study, the results in Fig. 3 have to be treated. More precisely, only the Mullins effect which occurs between the first and second loading cycles is considered. For this purpose, the data treatment proposed by Chagnon et al. [21] is considered: (i) the reloading path of the second cycle is assumed to coincide with the downloading path of the first cycle, (ii) the unloading data are horizontally shifted such that they start from zero strain and (iii) the unloading data are extended to rejoin the primary loading path. The comparison between original and corrected data is depicted in Fig. 4.

\section{Constitutive equations}

In this section, basics of hyperelasticity are first briefly recalled. From the pool of various available models, the neo-Hookean model is adopted due to its simplicity. Moreover, the pseudo-elastic model of Ogden and Roxburgh [17] is considered for describing the Mullins effect. The latter is modified and extended in order to account for the effect of swelling level.

\subsection{Hyperelasticity of swollen elastomers}

Consider swollen rubber material as a homogeneous, isotropic, hyperelastic and incompressible material. The body occupies a region $\Omega$ in its natural (undeformed and unstressed) configuration. Consider a material point $\mathbf{X}$ whose components in Cartesian coordinates are $X_{j}$ $(j=1,2,3)$. Due to the deformation process, the body occupies a new region $\Omega_{d}$, and the point $\mathbf{X}$ is moved to the spatial position $\mathrm{x}$ whose components are $x_{i}(i=1,2,3)$. The deformation gradient tensor, $\mathbf{F}$, is defined by

$\mathbf{F}=\operatorname{Grad} x=\frac{\partial x}{\partial X}$.

The left Cauchy-Green strain tensor is then simply given by $\mathbf{B}=\mathbf{F F}^{T}$. The response of hyperelastic materials is 

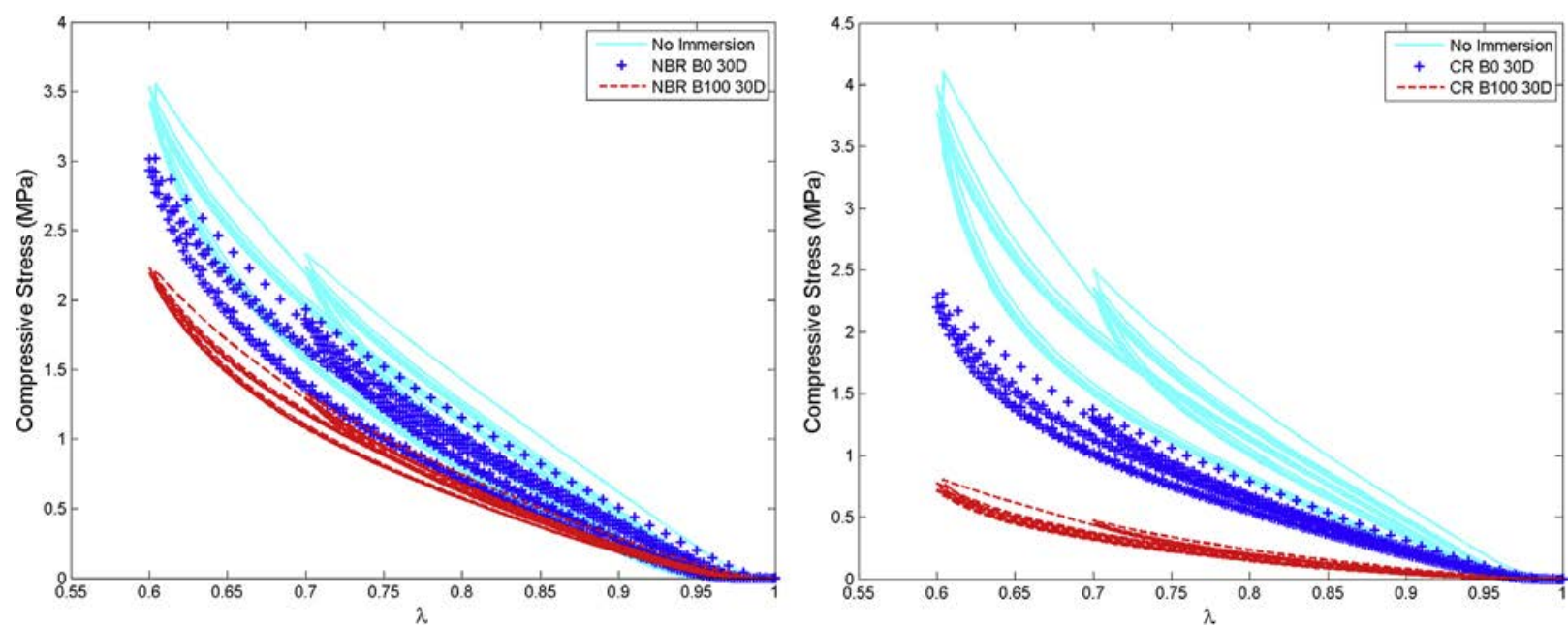

Fig. 3. Stress-strain curves of NBR (left) and CR (right) at dry (without immersion) and swollen states (after 30 days immersion in B0 and B100). For immersed rubbers, the stress is expressed with respect to the unswollen-unstrained configuration (initial dry cross section).

characterized by the existence of a strain energy function $W$ which depends on F. Considering the objectivity principle and isotropy, the form of $W$ for incompressible swollen elastomers can be expressed as a function of the two invariants of $\mathbf{B}$ and the degree of swelling $J_{s}$, i.e.:

$W=W\left(I_{1}, I_{2}, J_{s}\right)$.

where:

$I_{1}=\operatorname{tr} \mathbf{B} ; \quad I_{2}=\frac{1}{2}\left(I_{1}^{2}-\operatorname{tr} \mathbf{B}^{2}\right)$.

Considering the second law of thermodynamics, for a given (constant) degree of swelling, it can be shown that the Cauchy stress tensor is given by [22]:

$$
\begin{aligned}
\boldsymbol{\sigma}= & -p \mathbf{I}+2\left[\frac{\partial W\left(I_{1}, I_{2}, J_{s}\right)}{\partial I_{1}}+I_{1} \frac{\partial W\left(I_{1}, I_{2}, J_{s}\right)}{\partial I_{2}}\right] \mathbf{B} \\
& -2 \frac{\partial W\left(I_{1}, I_{2}, J_{s}\right)}{\partial I_{2}} \mathbf{B}^{2} .
\end{aligned}
$$

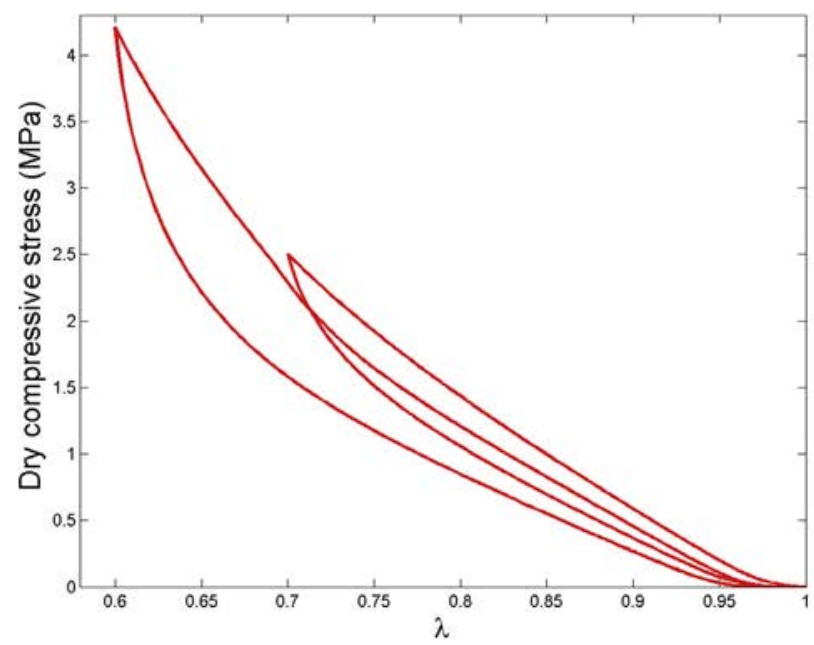

where $p$ is a Lagrange multiplier due to the assumption of incompressibility. It can be determined from the equilibrium equations and appropriate boundary conditions. It can be noted that the corresponding assumption is motivated by the experimental observation that swollen rubber specimens used in the present study undergo volume change of less than $5 \%$ during the mechanical tests.

\subsection{Brief recall on the pseudo-elastic model for Mullins effect in dry elastomers}

The basic assumption used in the pseudo-elastic model is the description of material response by the introduction of an additional continuous scalar parameter $\eta$ into the classical hyperelastic strain energy function for incompressible materials as follow [17]:

$$
\ddot{W}=\ddot{W}\left(I_{1}, I_{2}, \eta\right) \text {. }
$$

Due to the nature of $\eta$, the authors regarded $W$ as the pseudo-energy function and the ensuing constitutive

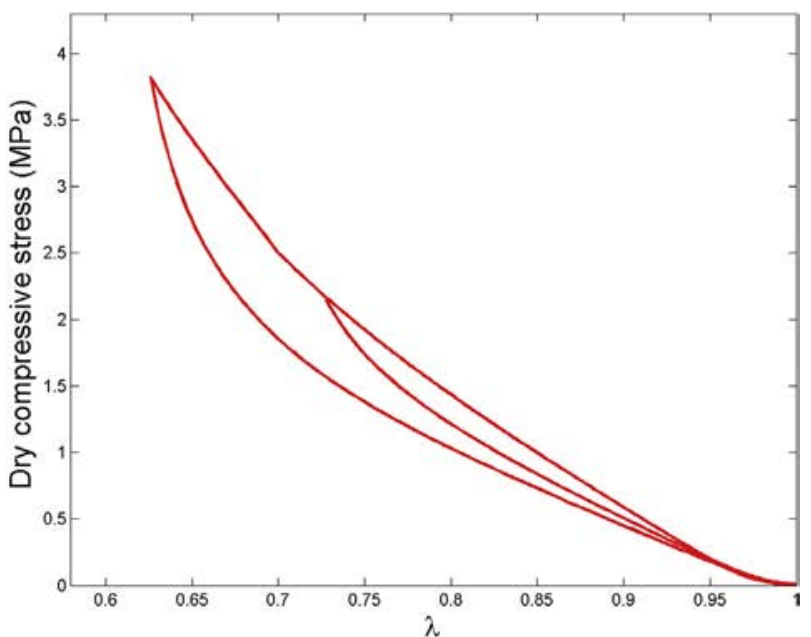

Fig. 4. Experimental results (left) and corrected data (right) for dry CR under cyclic compressive test. 
theory as pseudo-elasticity. In the context of rubber where the Mullins effect is considered as a damage phenomenon, the parameter $\eta$ can be related to the damage in the materials. During loading to a new maximum deformation state, the authors assumed that the damage stays zero. In this case, $\eta=1$ and the material response follows the classical undamaged (virgin) hyperelastic response. During unloading and subsequent reloading to submaximal deformation, $\eta$ evolves and its value depends on the current deformation state. In this case, the material response is described by another energy function as follow:

$\ddot{W}=\ddot{W}\left(I_{1}, I_{2}, \eta\left(I_{1}, I_{2}\right)\right)=\eta\left(I_{1}, I_{2}\right) \cdot W\left(I_{1}, I_{2}\right)$.

Using $W$ as a measure of deformation state, the authors showed that the parameter $\eta$ is given by:

$\eta=1-d$ where $d=\frac{1}{r} \operatorname{erf}\left[\frac{1}{m}\left(W_{\max }-W\left(I_{1}, I_{2}\right)\right)\right]$.

In the above expression, $d$ is the damage function, and $r$ and $m$ are material parameters. $r$ corresponds to a measure of the extent of the damage relative to the virgin state while $m$ controls the dependence of the damage on the extent of deformation. $W_{\max }$ is the value of $W$ at the maximum deformation state ever experienced during the loading history. For our purpose, which is detailed in Section 4.1, the role of parameters $r$ and $m$ are investigated by plotting $d$ for various values of $r$ and $m$, as depicted in Fig. 5. It is observed that $m$ dictates the initial slope of $d$ while $r$ simply scales vertically the curve $d$ to higher or lower values. More precisely, the initial slope of $d$ is an increasing function of $1 / \mathrm{m}$ (decreasing function of $m$ ) and the maximum value of $d$ is an increasing function of $1 / r$ (decreasing function of $r$ ).

\subsection{Extension of the pseudo-elastic model to Mullins effect in swollen elastomers}

In the case of swollen rubbers, the parameters $r$ and $m$ depend on the degree of swelling $J_{s}$ and the nature of interaction between rubber and solvent. In order to investigate the dependence of $r$ and $m$ on the swelling using experimental data, the damage function $d$ in Eq. (9) is phenomenologically modified and extended as follow:
Table 1

Flory-Huggins interaction parameter $\chi$ of each rubber-fuel system.

\begin{tabular}{ll}
\hline$\chi_{C R-B 100}$ & 0.311 \\
$\chi_{C R-B 0}$ & 1.356 \\
$\chi_{N B R-B 100}$ & 1.285 \\
$\chi_{N B R-B 0}$ & 1.767 \\
\hline
\end{tabular}

$d=d\left(J_{s}, \chi, I_{1, \max }-I_{1}\right)=\frac{1}{r\left(J_{s}, \chi\right)} \operatorname{erf}\left[\frac{1}{m\left(J_{s}, \chi\right)}\left(I_{1, \max }-I_{1}\right)\right]$.

In this expression, $\chi$ is the dimensionless interaction parameter describing the interaction between rubber and solvents, i.e. palm biodiesel and diesel. Note that $W$ has been replaced by $I_{1}=\operatorname{tr} \mathbf{B}$ as the measure of deformation state. $I_{1, \max }$ is the value of $I_{1}$ at the maximum deformation state ever experienced during the loading history. In this framework, during unloading and subsequent reloading to submaximal deformation, the Cauchy stress tensor in Eq. (6) becomes:

$$
\begin{aligned}
\boldsymbol{\sigma}= & -p \mathbf{I}+2(1-d)\left[\frac{\partial W\left(I_{1}, I_{2}, J_{s}\right)}{\partial I_{1}}+I_{1} \frac{\partial W\left(I_{1}, I_{2}, J_{s}\right)}{\partial I_{2}}\right] \mathbf{B} \\
& -2 \frac{\partial W\left(I_{1}, I_{2}, J_{s}\right)}{\partial I_{2}} \mathbf{B}^{2} .
\end{aligned}
$$

The choice of $W$ and the proposed explicit forms of $r\left(J_{s}, \chi\right)$ and $m\left(J_{s}, \chi\right)$ are discussed in Section 4.1.

\subsection{Special case of uniaxial compression}

For our purpose, we focus attention on the case of swollen elastomers under uniaxial compression. In this case, the deformation gradient tensor reduces to:

$\mathbf{F}=\lambda e_{1} \otimes e_{1}+\frac{1}{\sqrt{\lambda}}\left(e_{2} \otimes e_{2}+e_{3} \otimes e_{3}\right)$.

where $\lambda$ is the compression relative to the swollen-unstrained state of elastomeric specimens. Applying boundary conditions $\sigma_{22}=\sigma_{33}=0$ to determine $p$, the Cauchy stress along the direction of compression, is given by:

$\sigma=2(1-d)\left(\lambda^{2}-\frac{1}{\lambda}\right)\left(\frac{\partial W\left(I_{1}, I_{2}, J_{s}\right)}{\partial I_{1}}+\frac{1}{\lambda} \frac{\partial W\left(I_{1}, I_{2}, J_{s}\right)}{\partial I_{2}}\right)$.
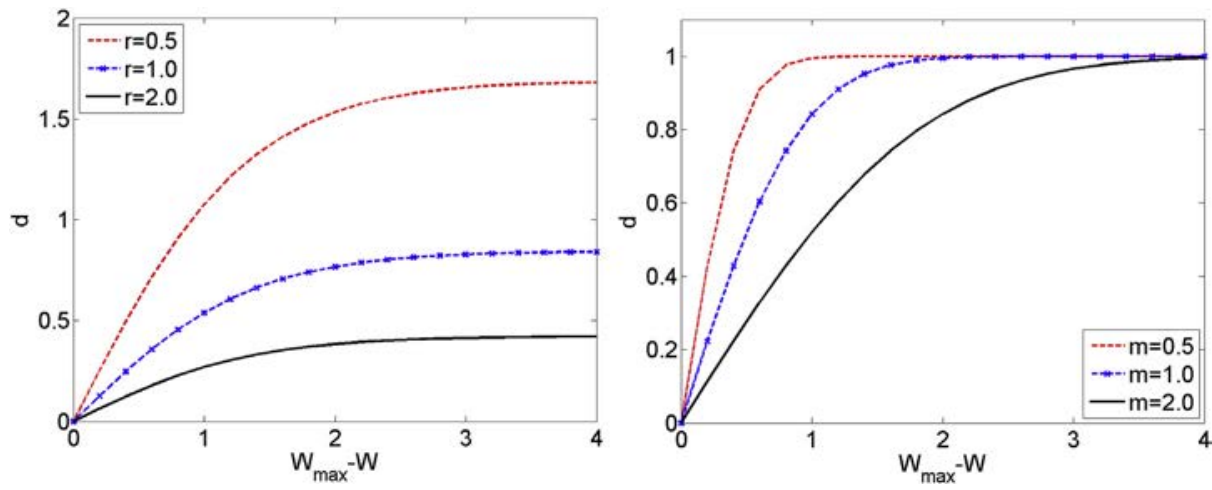

Fig. 5. Evolution of $\mathrm{d}$ as a function of $W_{\max }-W$ for $m=1$ and different values of $r$ (left) and for $r=1$ and different values of $m$ (right). 

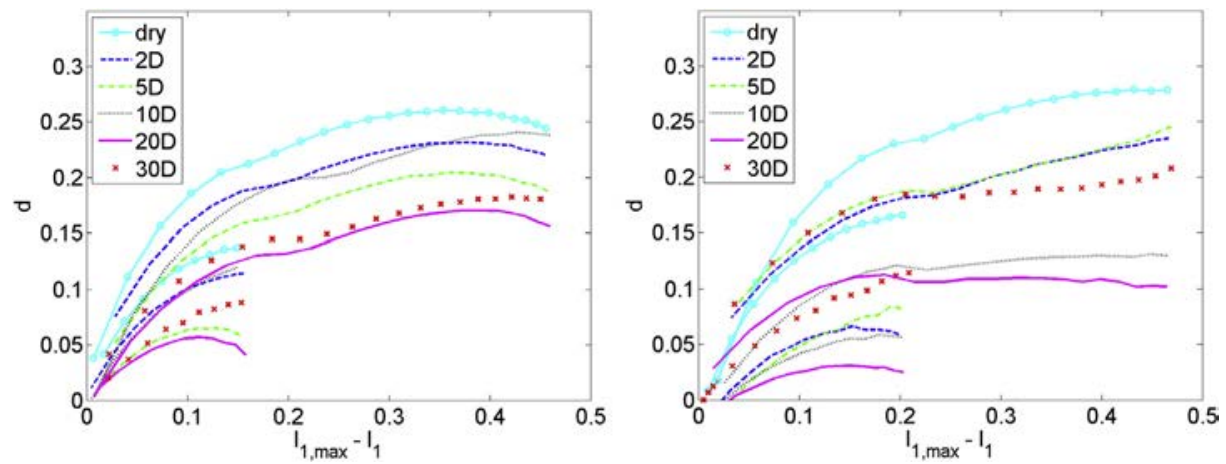

Fig. 6. Evolution of $d$ in CR swollen by B0 (left) and B100 (right). Results correspond to 2, 5, 10, 20 and 30 days of immersion duration. The short and long curves correspond to the maximum compressive strains of $30 \%(\lambda=0.7)$ and $40 \%(\lambda=0.6)$ respectively.
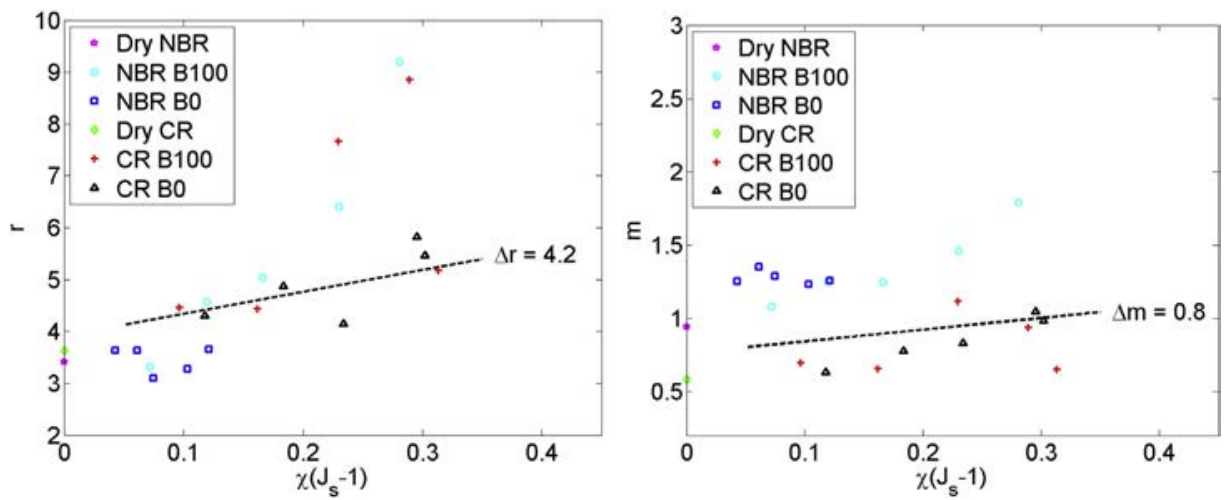

Fig. 7. Evolution of $r$ (left) and $m$ (right) as a function of $\chi\left(J_{s}-1\right)$. Results correspond to the maximum compressive strain of $40 \%$.

and the corresponding first Piola-Kirchhoff (engineering) stress relative to the swollen-unstrained area is

$P_{11}=2(1-d)\left(\lambda-\frac{1}{\lambda^{2}}\right)\left(\frac{\partial W\left(I_{1}, I_{2}, J_{s}\right)}{\partial I_{1}}+\frac{1}{\lambda} \frac{\partial W\left(I_{1}, I_{2}, J_{s}\right)}{\partial I_{2}}\right)$

\section{Results and discussion}

\subsection{Form of material functions}

In order to describe the general response of swollen rubbers, a simple neo-Hookean hyperelastic strain energy density is retained:

$W=\frac{\mu_{s}}{2}\left(I_{1}-3\right)$.

where $\mu_{S}$ is the shear modulus of swollen rubber. According to Treloar [19], swelling in rubber is a purely mixing or interdiffusion process with no chemical attraction between rubber and liquid molecules. Furthermore, the only effect of swelling is to reduce the modulus in inverse proportion to

Table 2

Summary of material parameters required in the proposed model.

\begin{tabular}{llll}
\hline Hyperelasticity (neo-Hookean) & $\mu_{d}$ & & \\
Mullins effect & $r_{d}$ & $m_{d}$ & \\
Swelling & $n$ & $\Delta r$ & $\Delta m$ \\
\hline
\end{tabular}

the cube root of the degree of swelling without changing the form of the stress-strain curve. Generalizing this idea, the shear modulus of the swollen rubber $\mu_{s}$ is assumed to be related to that of dry rubber $\mu_{d}$ through:

$\mu_{s}=J_{s}^{-n} \mu_{d}$.

It follows that the strain energy function of the swollen rubber becomes

$W=J_{s}^{-n} \frac{\mu_{d}}{2}\left(I_{1}-3\right)$

where $n$ is a parameter to be determined from all sets of experimental data. In this case, the engineering stress in Eq. (14) reduces to:

$P_{11}=J_{s}^{-n} \mu_{d}(1-d)\left(\lambda-\frac{1}{\lambda^{2}}\right)$.

where $d$ is given by Eq. (10).

Table 3

Values of material parameters used in the model.

\begin{tabular}{llll}
\hline & NBR & CR \\
\hline$\mu_{d}(\mathrm{MPa})$ & 1.7786 & & 1.9102 \\
$r_{d}$ & 4.4340 & & 3.4182 \\
$m_{d}$ & 0.272 & & 0.1683 \\
$n$ & & 2.5 & \\
$\Delta r$ & & 4.2 & \\
$\Delta m$ & & 0.8 & \\
\hline
\end{tabular}



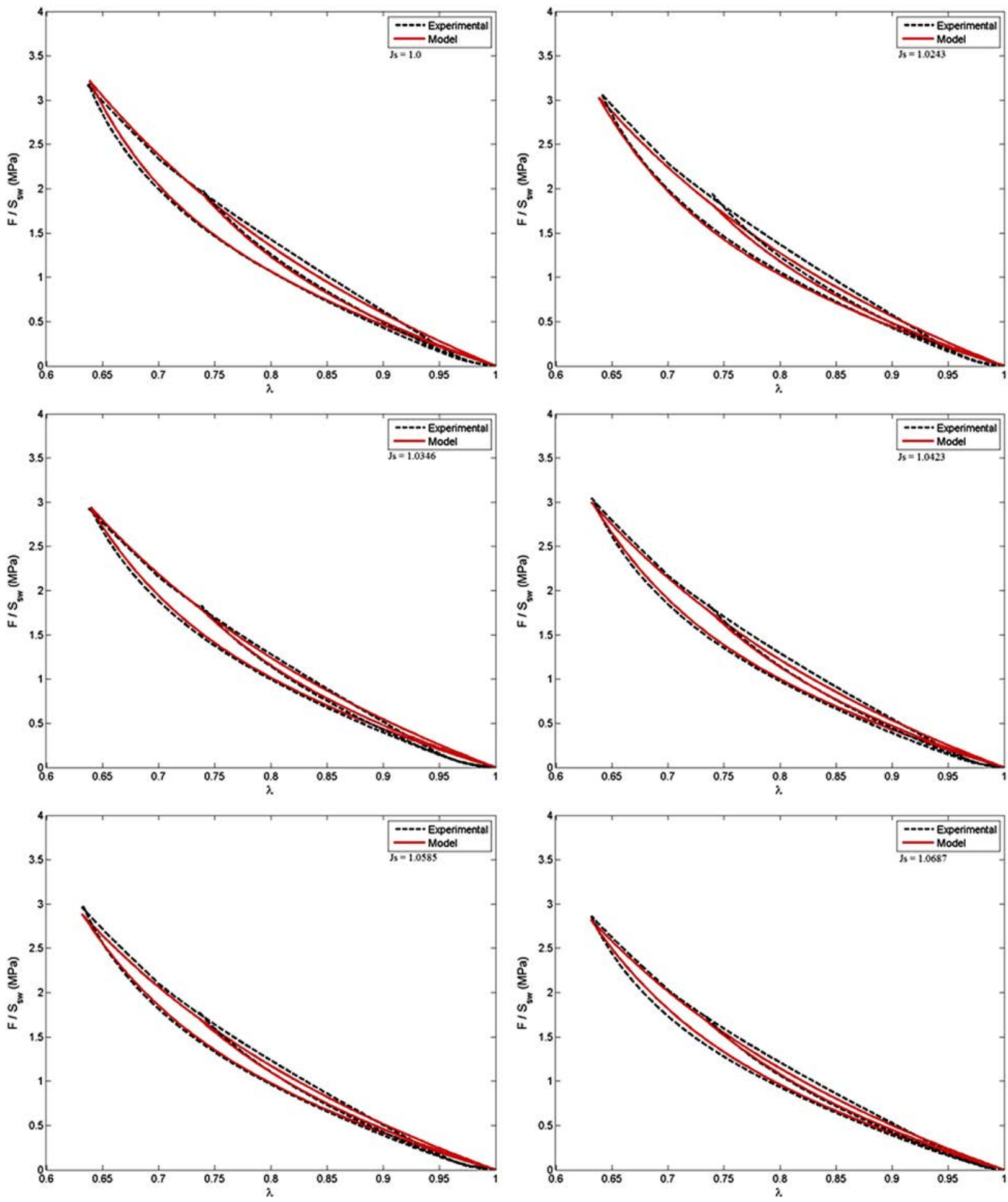

Fig. 8. Comparison between model and experiment for dry NBR and NBR swollen by B0 ( $\chi=1.7669)$.

Before proceeding further, it is necessary to determine the values of the dimensionless interaction parameter $\chi$ describing the interaction between rubbers (CR and NBR) and solvents (B0 and B100). The corresponding parameter can be obtained using the classical Flory-Huggins equation by assuming that equilibrium swelling is reached after 30 days of immersion. By considering the properties of B0 and B100 used in the present study, the following parameters are obtained without further details (see Table 1). 

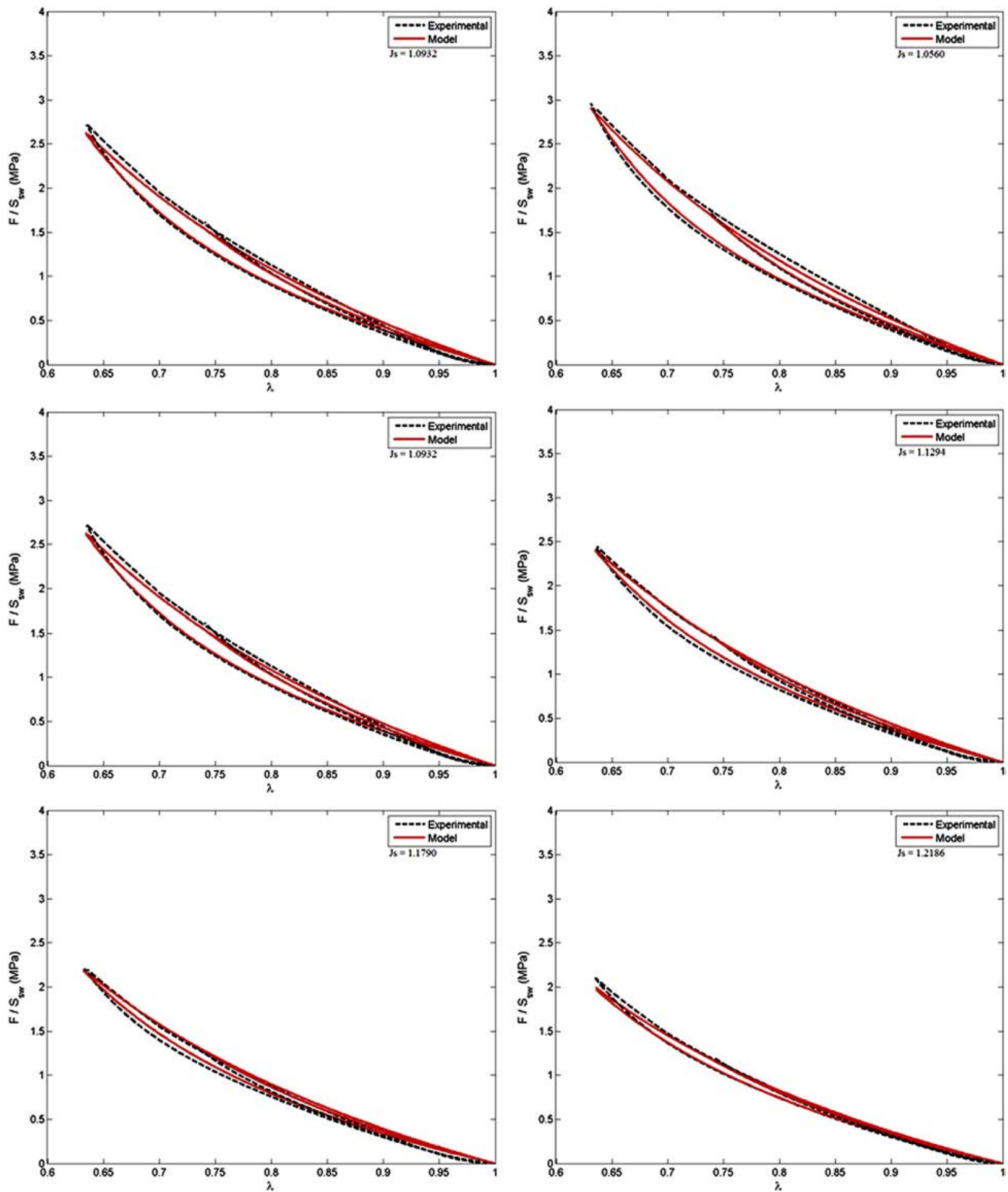

Fig. 9. Comparison between model and experiment for dry NBR and NBR swollen by B100 $(\chi=1.2855)$.

In order to determine the explicit forms of $r\left(J_{s}, \chi\right)$ and $m\left(J_{s}, \chi\right)$, the treated experimental data like the one shown in Fig. 4 (right) are considered. More precisely, the experimental values of damage $d$ are plotted as a function of $I_{1, \max }-I_{1}$. The results for CR swollen by B0 and B100 are depicted in Fig. 6. Note that the corresponding results for NBR swollen by B0 and B100 are not shown since they are qualitatively similar. In Fig. $6, d$ is obtained from the following: 

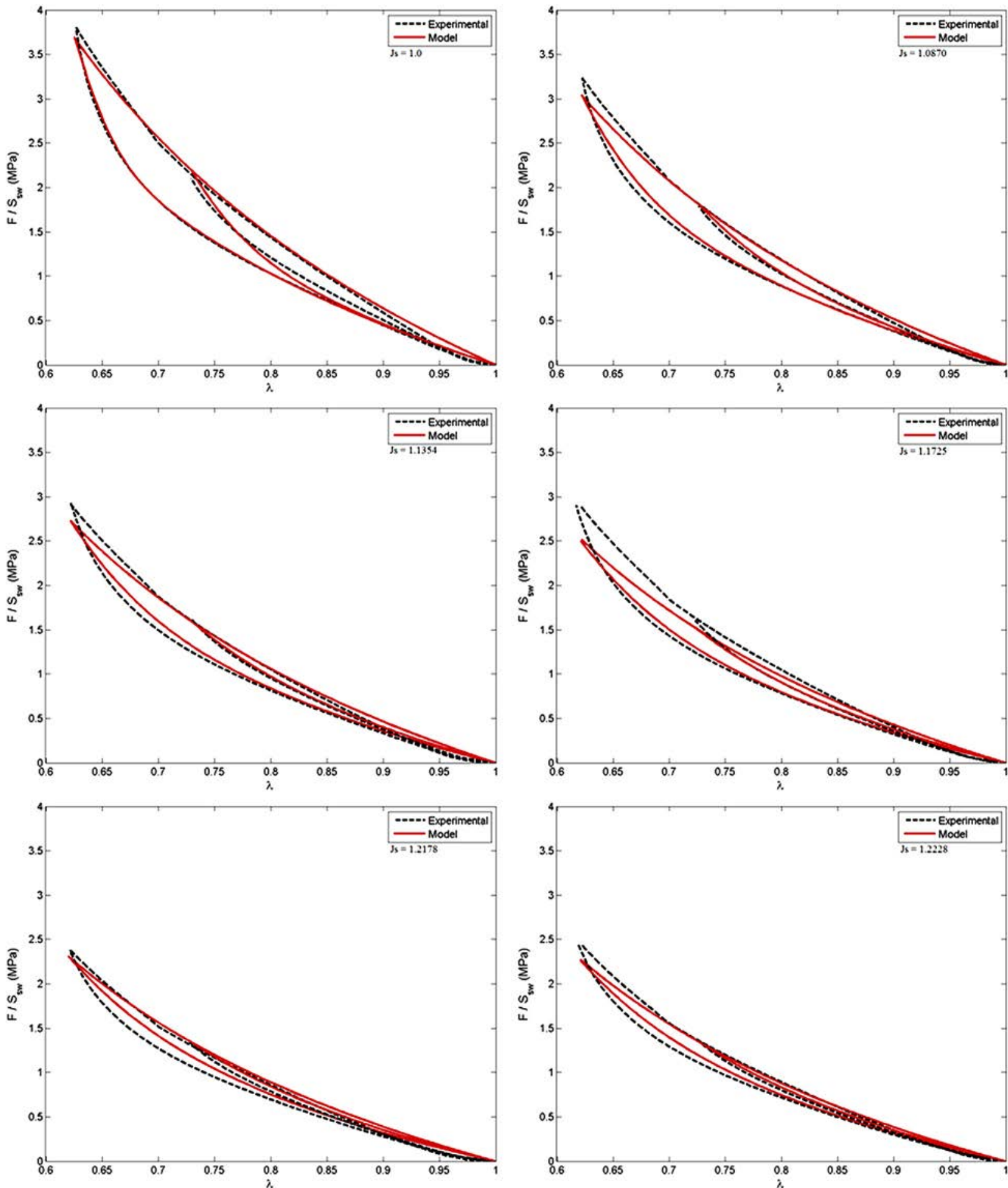

Fig. 10. Comparison between model and experiment for dry CR and CR swollen by B0 ( $\chi=1.3561)$.

$d(d r y)=\frac{P_{d 1}^{u p}-P_{d 1}^{u n}}{P_{d 1}^{u p}} ; \quad d($ swollen $)=\frac{P_{s 1}^{u p}-P_{s 1}^{u n}}{P_{s 1}^{u p}}$

where $P_{d 1}^{u p}$ is the stress in dry rubber during loading of the first cycle, $P_{d 1}^{u n}$ is the stress in dry rubber during unloading of the first cycle, $P_{s 1}^{u p}$ is the stress in swollen rubber during loading of the first cycle and $P_{s 1}^{u n}$ is the stress in swollen rubber during unloading of the first cycle. It is generally observed that the damage is strongly affected by the degree of swelling and the type of rubber-solvent, i.e. interaction 

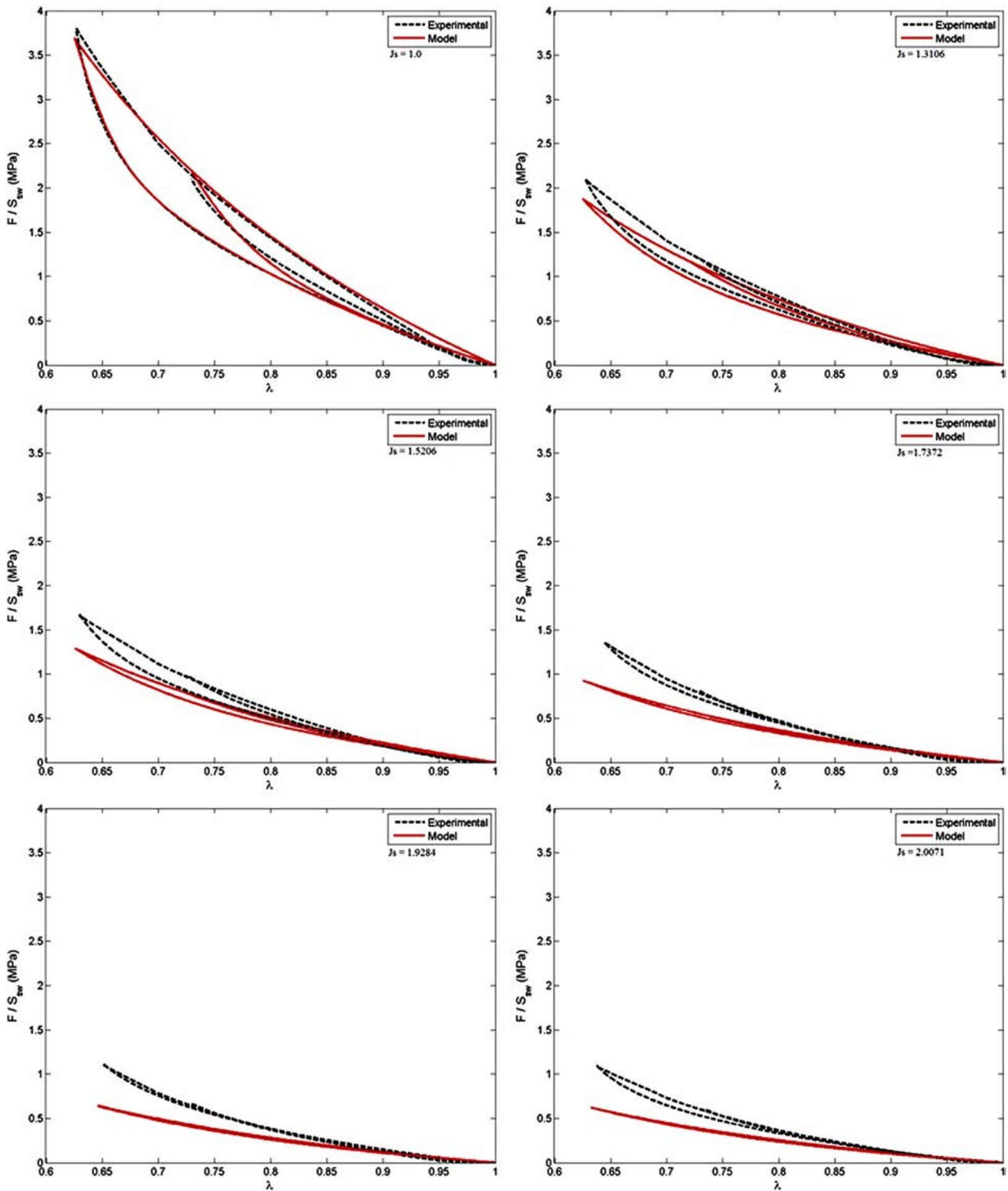

Fig. 11. Comparison between model and experiment for dry $C R$ and $C R$ swollen by $B 100(\chi=0.3113)$.

between rubber and biodiesel/diesel. More precisely, the Mullins effect appears to decrease with the increase of degree of swelling.

By considering the initial slope and the maximum value of $d$ in Fig. 6, it is possible to plot the evolution of both $m$ and $r$ as a function of, $\chi\left(J_{s}-1\right)$ as shown in Fig. 7. In general, it is observed that both $r$ and $m$ are increasing functions of $\chi\left(J_{s}-1\right)$. Motivated by this finding, and for the sake of simplicity, the parameters $r$ and $m$ are assumed to evolve in a simple linear way as follow: 
$r=r_{d}+\Delta r \chi\left(J_{s}-1\right) ; \quad m=m_{d}+\Delta m \chi\left(J_{s}-1\right)$.

where $r_{d}$ and $m_{d}$ are the values of $r$ and $m$ for dry rubbers. $\Delta r$ and $\Delta m$ are additional material constants. Finally, the damage function $d$ in Eq. (10) becomes:

$d=\frac{1}{r_{d}+\Delta r \chi\left(J_{s}-1\right)} \operatorname{erf}\left[\frac{1}{m_{d}+\Delta m \chi\left(J_{s}-1\right)}\left(I_{1, \max }-I_{1}\right)\right]$.

To summarize, the following parameters in Table 2 have to be identified.

\subsection{Identification of material parameters}

In the following, methods to estimate material parameters of the model are discussed. The parameter $\mu_{d}$ can be obtained by fitting the primary curve of dry rubber using neo-Hookean hyperelastic strain energy, while $r_{d}$ and $m_{d}$ can be identified from the set of secondary curves (downloading) of dry rubber. The power law parameter $n$, can be estimated by considering the primary curve of dry rubber and primary curves of corresponding swollen rubbers having different degrees of swelling. The parameters $\Delta r$ and $\Delta m$ can be estimated by considering the slopes of the data in Fig. 7 left and right, respectively. Since the slopes of $r$ and $m$ do not vary significantly for different rubber-solvent combinations, the values of $\Delta r$ and $\Delta m$ are assumed to be independent of the type of rubber and solvent, and their estimated values are illustrated by dashed lines. Finally, the estimated parameters are summarized in Table 3.

\subsection{Comparison between model and experiment}

The ability of the proposed model to describe the Mullins effect in swollen rubbers under cyclic compressive loading conditions is presented in Figs. 8-11. As a comparison, results related to the dry rubber are also shown in each figure.

In general, it is observed that the proposed model is qualitatively in good agreement with experiments. The primary curves of dry and swollen rubbers are welldescribed. Moreover, the effects of swelling on the stresssoftening due to the Mullins effect are well-predicted. Some discrepancies between model and experiments are found for swollen rubbers having relatively high degree of swelling. Indeed, as shown in Figs. 9 and 10, the model appears to slightly underestimate the stress level in highly swollen rubbers. Furthermore, for the case of CR swollen by B100, the stress level and Mullins effect predicted by the model are significantly smaller than the one obtained from the experiments, as presented in Fig. 11. The corresponding discrepancies could be attributed to the value of parameters $\Delta r$ and $\Delta m$ used in the present model. Indeed, for the sake of simplicity, they are chosen to be independent of the rubber type and rubber-solvent combination. Furthermore, it was also assumed that Treloar's theory holds for all swollen rubbers [19], i.e. no chemical attraction between rubber and solvent molecules in swollen rubbers. While this assumption appears to be acceptable for relatively low degree of swelling, its validity in the case of highly swollen rubber such as CR in B100 becomes questionable. Indeed, as discussed in [1], chemical reaction and interaction occurs between the B100 molecules and CR backbone resulting to the degradation of its mechanical properties.

\section{Conclusions}

In this work, experimental investigation and modelling of the Mullins effect in dry and swollen rubbers were addressed. For this purpose, a set of mechanical tests were conducted in order to probe the Mullins effect in dry and swollen rubbers under cyclic loading conditions. Two types of filled rubber were considered: NBR and CR. The swollen rubbers were obtained by immersing them into different solvents, i.e. palm biodiesel and diesel, for various immersion times. It was shown that both dry and swollen rubbers exhibit inelastic responses such as the Mullins effect, hysteresis and permanent set. More precisely, it was found that the Mullins effect decreases as the degree of swelling increases. To describe this observation, the pseudo-elastic model of Ogden and Roxburgh (1999) was considered and modified in order to account for the swelling level. Results showed that, in general, the proposed model was qualitatively in good agreement with experimental observations.

It should be noted that the present works focuses only on the Mullins effect. Further investigations on the other inelastic responses such as permanent set and hysteresis in swollen rubber are needed to improve the proposed model.

\section{Acknowledgements}

The authors greatly appreciate the financial support from Ministry of Higher Education Malaysia through High Impact Research Grant HIR-MOHE D000008-16001 and the support from University of Malaya through Postgraduate Research Grant PV048/2011B.

\section{References}

[1] A.S.M.A. Haseeb, T.S. Jun, M.A. Fazal, H.H. Masjuki, Degradation of physical properties of different elastomers upon exposure to palm biodiesel, Energy 36 (2011) 1814-1819.

[2] W. Trakarnpruk, S. Porntangjitlikit, Palm oil biodiesel synthesized with potassium loaded calcined hydrotalcite and effect of biodiesel blend on elastomer properties, Renew. Energ. 33 (2008) 1558-1563.

[3] A. Andriyana, N. Saintier, E. Verron, Configurational mechanics and critical plane approach: concept and application to fatigue failure analysis of rubberlike materials, Int. J. Fatigue 32 (2010) 1627-1638.

[4] E. Verron, A. Andriyana, Definition of a new predictor for multiaxial fatigue crack nucleation in rubber, J. Mech. Phys. Solids 56 (2008) 417-443.

[5] G. Marckmann, E. Verron, Comparison of hyperelastic models for rubber-like materials, Rubber Chem. Technol. 79 (2006) 835-858.

[6] J.S. Bergström, M.C. Boyce, Constitutive modelling of the large strain time-dependent behavior of elastomers, J. Mech. Phys. Solids 46 (1998) 931-954.

[7] A. Lion, On the large deformation behaviour of reinforced rubber at different temperatures, J. Mech. Phys. Solids 45 (1997) 1805-1834.

[8] S. Trabelsi, P. Albouy, J. Rault, Crystallization and melting processes in vulcanized stretched natural rubber, Macromolecules 36 (2003) 7624-7639.

[9] H. Bouasse, Z. Carriére, Courbes de traction du caoutchouc vulcanisé, Ann. Fac. Sci. Toulouse 5 (1903) 257-283.

[10] L. Mullins, Effect of stretching on the properties of rubber, Rubber Chem. Technol. 21 (1948) 281-300.

[11] J. Diani, B. Fayolle, E. Gilormini, A review on the Mullins effect, Eur. Polym. J. 45 (2009) 601-612. 
[12] A. Andriyana, A.B. Chai, E. Verron, M.R. Johan, Interaction between diffusion of palm biodiesel and large strain in rubber: effect on stress-softening during cyclic loading, Mech. Res. Commun. 43 (2012) 80-86.

[13] A.B. Chai, A. Andriyana, E. Verron, M.R. Johan, Mechanical characteristics of swollen elastomers under cyclic loading, Mater. Des. 44 (2013) 566-572.

[14] G. Marckmann, E. Verron, L. Gornet, G. Chagnon, P. Charrier, P. Fort, A theory of network alteration for the Mullins effect, J. Mech. Phys. Solids 50 (2002) 2011-2028.

[15] W.C. Lin, W. Fan, A. Marcellan, D. Hourdet, C. Creton, Large strain and fracture properties of poly(dimethylacrylamide)/silica hybrid hydrogels, Macromolecules 43 (2010) 2554-2563.

[16] R.E. Webber, C. Creton, H.R. Brown, J.P. Gong, Large strain hysteresis and Mullins effect of tough double-network hydrogels, Macromolecules 40 (2007) 2919-2927.
[17] R.W. Ogden, D.G. Roxburgh, A pseudo-elastic model for the Mullins effect in filled rubber, Proc. Roy. Soc. A - Math. Phy. 455 (1999) 2861-2877.

[18] A.B. Chai, A. Andriyana, E. Verron, M.R. Johan, A.S.M.A. Haseeb, Development of a compression test device for investigating interaction between diffusion of biodiesel and large deformation in rubber, Polym. Test. 30 (2011) 867-875.

[19] L. Treloar, The Physics of Rubber Elasticity, Oxford University Press, London, 1975.

[20] A.N. Gent, Engineering with Rubber. How to Design Rubber Components, second ed., Hanser, 2001.

[21] G. Chagnon, On the relevance of Continuum Damage Mechanics as applied to the Mullins effect in elastomers, J. Mech. Phys. Solids 52 (2004) 1627-1650.

[22] G.A. Holzapfel, Nonlinear Solid Mechanics: A Continuum Approach for Engineering, John Wiley \& Sons Ltd., 2000. 